\title{
Methodik und was kann die Nervensonografie
}

\author{
Methodology and Scope of Sonography of the Nerves
}

\author{
Autor \\ T. Schelle \\ Institut \\ Klinik für Neurologie, Städtisches Klinikum Dessau-Roßlau
}

\author{
Schlüsselwörter \\ - hochauflösender Ultraschall \\ peripherer Nerv \\ - Engpasssyndrome \\ - Nerventrauma \\ - periphere Nervenscheiden- \\ tumore \\ - Polyneuropathie \\ Key words \\ - high-resolution ultrasound \\ - peripheral nerve \\ - entrapment-syndrome \\ - injuries of the peripheral \\ nerves \\ tumours of the peripheral \\ nerve sheath \\ polyneuropathies
}

Bibliografie

DOI http://dx.doi.org/

$10.1055 / \mathrm{s}-0035-1549945$

Klin Neurophysiol 2015;

46: 79-89

(c) Georg Thieme Verlag KG

Stuttgart · New York

ISSN 1434-0275

Korrespondenzadresse

Dr. Thomas Schelle

Klinik für Neurologie

Städtisches Klinikum

Dessau-Roßlau

Auenweg 38

06847 Dessau-Roßlau

thomas.schelle@klinikum-

dessau.de

\section{Zusammenfassung}

Bildgebende Untersuchungen gewinnen bei der Diagnostik von/Erkrankungen peripherer Nerven zunehmend an Bedeutung. Die hochauflösende Sonografie und das MRT liefern dabei wichtige morphologische Informationen über Nervenstämme, Nervenwurzeln und den Plexus brachialis. Im Gegensatz zur elektrophysiologischen Untersuchung ermöglichen sie die millimetergenaue Lokalisation der Läsion und geben in vielen Fällen auch Auskunft über deren Ätiologie. Während der letzten Jahre wurden zahlreiche Artikel über die Anwendung des hochauflösenden Ultraschalls bei der Diagnostik von Engpasssyndromen, traumatischen Nervenverletzungen, Raumforderungen am peripheren Nerven und Polyneuropathien veröffentlicht. Diese Arbeit versucht unter Berücksichtigung der persönlichen Erfahrungen des Autors einen Überblick über die wichtigsten Entwicklungen auf diesem Feld zu geben.

\section{Einführung \\ $\nabla$}

Mehr als 2 Jahrzehnte nach der Erstbeschreibung der sonografischen Darstellung peripherer Nerven bzw. von deren pathologischen Veränderungen im MRT haben beide Methoden eine zunehmende Bedeutung in der klinischen Routine erlangt [1-4]. Diese Tatsache spiegelt sich auch in der wachsenden Zahl an publizierten wissenschaftlichen Arbeiten wieder. Als komplementäre Untersuchungen liefern sie wichtige Informationen über die Morphologie des peripheren Nervensystems, die exakte Lokalisation von pathologischen Prozessen und in vielen Fällen auch über die Ursache einer Nervenläsion. Beide Verfahren ersetzen jedoch keinesfalls die genaue Anamneseerhebung und klinische Untersuchung und dienen daher genauso wie die Elektrophysiologie hauptsächlich zur Sicherung der vermu-

\section{Abstract \\ $\nabla$}

Imaging-techniques of the peripheral nervous system play an increasing role in the diagnosis of peripheral nerve disorders. High-resolution ultrasound and magnetic resonance imaging provide significant information about the morphology of the peripheral nerves, nerve-roots and the brachial plexus. In contrast to the nerve conduction studies, they allow a more precise localisation of the lesion as well as offer information about the underlying aetiology. During the past decade numerous papers about high-resolution ultrasound concerning applications for entrapment syndromes, traumatic nerve injuries, space-occupying lesions of the peripheral nerves and polyneuropathies have been published. This review presents an overview about the most important developments in this field taking into account the personal experiences of the author.

teten Diagnose. Kontinuierlich verbesserte Ultraschallgeräte und Linearsonden ermöglichen nicht nur die Abbildung sehr kleiner peripherer Nerven, zervikaler Nervenwurzeln und des Plexus brachialis, sondern auch die Anwendung des hochauflösenden Ultraschalls im Zusammenhang mit neuen Untersuchungsverfahren. Exemplarisch hierfür soll die Darstellung der Mikrovaskularisation (z.B. Superb Micro Vascular Imaging=SMI, Toshiba Medical Systems), der Gewebeperfusion nach Gabe von UltraschallKontrastverstärker (Contrast Enhanced Ultrasound $=$ CEUS) und schließlich die quantitative Steifigkeitsmessung von Geweben mittels Scherwellenelastometrie (z.B. Virtual Touch ${ }^{\mathrm{TM}}$ Tissue Quantification, Siemens AG) genannt werden. Besonders die letztgenannte Methode hat in Vorläuferstudien vielversprechende Ergebnisse bei der Diagnostik von Weichteilraumforderungen 
und Engpasssyndromen generiert [5,6]. Darüber hinaus eignet sich die Sonografie auch zur ultraschallgestützten Therapie neuropathischer Schmerzen (gepulste Radiofrequenz-Ablation, Kryoablation, periphere Nervenstimulation) und wird bereits seit längerer Zeit in der Regionalanästhesie eingesetzt [7-12]. Diese Übersichtsarbeit versucht den aktuellen Kenntnisstand über Methodik und Anwendungsmöglichkeiten der Nervensonografie zusammenzufassen.

\section{Untersuchungstechnik und Normalbefunde \\ $\nabla$}

In der Stellungnahme der Deutschen Gesellschaft für Ultraschall in der Medizin (DEGUM) wird als technischer Mindeststandart für die Nervensonografie ein Ultraschallsystem mit einem $10 \mathrm{MHz}$ Linearschallkopf empfohlen [13]. Schon damit erreicht man gute axiale und laterale Auflösungen von 0,2 vs. 0,6 mm [14]. Aktuelle Geräte werden meist mit Multifrequenzlinearsonden bis 18 (22) MHz ausgeliefert, womit sich das Auflösungsvermögen, wenn auch nicht proportional, weiter steigern lässt. Das hat allerdings zur Folge, dass aus physikalischen Gründen die Eindringtiefe des Ultraschalls im Gewebe mit zunehmender Sendefrequenz abnimmt [14]. Tief liegende Strukturen $(>5 \mathrm{~cm})$ können deshalb nicht mit voller Auflösung untersucht werden. Nach der Erfahrung des Autors sind Multifrequenzschallköpfe von den Firmen häufig nur auf den obersten Frequenzbereich optimiert, sodass es sinnvoll sein kann, sich mehrere Linearschallköpfe (z.B. 18 und $10 \mathrm{MHz}$ ) zuzulegen. Neben der richtigen Sondenauswahl ist das eingesetzte Bildbearbeitungsverfahren von entscheidender Bedeutung. Zur besseren Erkennbarkeit von Gewebegrenzen und zur Artefaktreduktion wird ein Echtzeit-Compound-Imaging verwendet, welches unter verschiedenen Winkeln aufgenommene Einzelscans desselben Objektes zu einem endgültigen Bild verrechnet [3,4]. Darüber hinaus bieten die unterschiedlichen Hersteller besonders bei High-End-Systemen weitere Verfahren zur Bildverbesserung an, auf die hier im Einzelnen nicht eingegangen wird. Eine weitere Steigerung der Abbildungsleistung lässt sich durch die Verwendung von Wasservorlaufstrecken (alternativ viel Ultraschallgel), durch einen streng senkrechten Insonationswinkel und durch die ständige Positionierung des elektronischen Fokus in Höhe des Untersuchungsobjektes erreichen $[3,4]$. Außerdem können in den Presets (Small-Parts) meist weitere Bildoptimierungen (z.B. dynamischer Bereich) vorgenommen werden. Das Aufsuchen der peripheren Nerven und Nervenwurzeln erfolgt durch die Landmarkentechnik, welche gute Kenntnisse in der sonografischen Schnittbildanatomie voraussetzt $[3,4]$. Einen Überblick über häufiger und weniger häufig untersuchte Nerven und deren Landmarken zum Aufsuchen geben die $\diamond$ Tab. 1a und $\diamond$ Tab. 1b [15-40]. Die weiter oben genannten Werte für das axiale und laterale Auflösungsvermögen erklären, warum sich mit derartigen Ultraschallsystemen einzelne Faszikel und das Hüllgewebe (Peri- und Epineurium), nicht jedoch einzelne Axone oder das Endoneurium sonografisch darstellen lassen. Folglich ähnelt im Querschnitt ein peripherer Nerv einer Honigwabe mit hypoechogenen Faszikeln und hyperechogenem Hüllgewebe, im Längsschnitt erinnert er eher an eine kabelartige Struktur $[41,42]$. Der Anteil an Hüllgewebe und die Zahl der Faszikel nimmt mit zunehmender Differenzierung von proximal nach distal zu [43]. Ein Beispiel zeigt $\odot$ Abb. 1. Neben den o.g. Hauptscanebenen, in denen alle krankhaften Veränderungen entweder als Einzelimage oder Videoloop dokumentiert werden sollten, lässt sich der periphe- re Nerv unter beliebigen anderen Insonationswinkeln untersuchen und auch dynamische Betrachtungen (aktive und passive Verschiebbarkeit) sind möglich [3,4]. Darüber hinaus können im B-Bild eine Reihe von quantitativen Messungen vorgenommen werden, wie z.B. die Bestimmung des Nervendurchmessers und der Nervenquerschnittsfläche (Cross Sectional Area=CSA). Letztere hat sich inzwischen allgemein als diagnostischer Standard etabliert und wird durch Umfahren der Grenze zwischen hypoechogenen Faszikeln und hyperechogenem Hüllgewebe bestimmt [29]. Von dieser Größe existieren eine ganze Reihe Derivate für die Diagnostik von Engpasssyndromen und Polyneuropathien (Swelling-Ratios, inter- und intranerve CSA - Variability), die sich in der klinischen Routine bisher nur zum Teil durchgesetzt haben [44-47]. Die Messung der Nervenquerschnittfläche weist eine gute intra- und interrater Reliabilität auf [27, 29]. In größeren prospektiven Normwertstudien wurden darüber hinaus einige Zusammenhänge der Nervenquerschnittsfläche mit verschiedenen demografischen Faktoren herausgearbeitet. Es existiert eine positive Korrelation mit dem Alter [47-51] und dem Body-Mass-Index [22,48,50,52]. Männer haben eine größere Nervenquerschnittsfläche als Frauen [29,47-49,51,53,54]. Einige Autoren berichten darüber hinaus über eine positive Korrelation mit Körpergröße [22,52,54] und -Gewicht [47,52,54]. Noch bedeutsamer, insbesondere bei Anwendung von „Cut-Off“ Werten für die Diagnostik von Engpasssyndromen aus asiatischen Studien auf unsere Patienten scheint die Tatsache, dass sich die Nervenquerschnittflächen dieser Populationen unterscheiden [55]: In Nordamerika und Europa weisen die Kontrollen am N. medianus größere physiologische Grenzwerte auf, als in Asien ( Tab. 2) [27, 29,53,55-58]. Diverse Normwerte können den Tab. 1a, b entnommen werden. Auf weitere Untersuchungsmöglichkeiten (Farbdoppler usw.) wird in den folgenden Unterabschnitten näher eingegangen.

\section{Anwendungsmöglichkeiten bei Engpasssyndromen $\nabla$}

Die sonografische Diagnostik von Engpasssyndromen, insbesondere des weitverbreiteten Karpaltunnelsyndroms (KTS), ist bisher am besten wissenschaftlich untersucht worden [59]. Dabei finden sich einige Merkmale, die bei den unterschiedlichen Krankheitsbildern gemeinsam vorkommen ( $\bullet$ Abb. 2). Sie sollen deshalb zuallererst besprochen werden: Die über einen längeren Zeitraum anhaltende externe Kompression eines peripheren Nerven durch unterschiedliche Noxen (Raumforderungen, Bänder, Knochen usf.) führt an der Kompressionsstelle zu einem Kalibersprung. Proximal, beim KTS häufig auch distal davon, findet man ein fokales Ödem mit Zunahme der Nervenquerschnittsfläche. Die Faszikel erscheinen hier hypoechogen aufgetrieben oder völlig maskiert [60]. Diese fokale Schwellung kann durch Messung (CSA) und Anwendung von „Cut-Off“ Werten zur Sicherung der Diagnose verwendet werden [61-64]. Alternativ lassen sich auch „Ratios“ durch Bildung des Quotienten dieses „CutOff“-Wertes mit der normalisierten Nervenquerschnittfläche proximal davon berechnen $[44,45]$. Für das KTS wurden aus nordamerikanischen, europäischen und asiatischen Populationen zahleiche „Cut-Off“ Werte publiziert, deren Anwendung zum Teil die Sensitivität von elektrophysiologischen Methoden erreicht, während in Metaanalysen eine gewisse Unterlegenheit des Ultraschalles gegenüber der Neurografie festgestellt worden ist. Einen Überblick gibt ○ Tab. 3 [44,61-63,65]. Dennoch hat die amerikanische Vereinigung für neuromuskuläre und elektro- 
Tab. 1a Nerven, anatomische Landmarken und Normwerte des CSA $\left(\mathrm{mm}^{2}\right)$ bzw. des Anterior-Posterioren Durchmessers (mm) \pm Standardabweichung Kopf, Hals und obere Extremität. ( $R=$ Range), [15-40].

\begin{tabular}{|c|c|c|c|}
\hline Nerv & Landmarke & Normwerte & Referenzen \\
\hline N. vagus & Vagina carotica zwischen $\mathrm{A}$. carotis communis und V. jugularis interna & ? & Le Corroller T et al. 2009 \\
\hline N. accessorius & $\begin{array}{l}\text { Austritt aus dem Hinterrand des M. sternocleidomastoideus oder Eintritt in den } \\
\text { Vorderrand des M. trapezius }\end{array}$ & $0,76 \pm 0,12 \mathrm{~mm}$ & Mirjalili SA et al. 2012 \\
\hline N. phrenicus & Auf dem Vorderrand des M. scalenus anterior, A. cervicalis ascendens & $?$ & Canella C et al. 2010 \\
\hline $\begin{array}{l}\text { N. auricularis } \\
\text { magnus }\end{array}$ & $\begin{array}{l}\text { Zwischen Platysma und M. sternocleidomastoideus, ventral am „Punctum } \\
\text { nervosum“ }\end{array}$ & $1,4 \pm 0,30 \mathrm{~mm}$ & Lieba-Samal D et al. 2014 \\
\hline $\begin{array}{l}\text { Nn. supraclavicu- } \\
\text { lares }\end{array}$ & Unter Hinterrand des M. sternocleidomastoideus, „Punctum nervosum“ & $?$ & Herring AA et al. 2012 \\
\hline $\begin{array}{l}\text { Rami ventrales } \\
\text { C5-C8 }\end{array}$ & $\begin{array}{l}\text { Interscalenärer Raum (M. scalenus anterior und -medius), Lokalisation durch } \\
\text { Anzahl d. Tubercula der Processus transversus: } \\
\text { C5 und C6-oberfl. Lage in der interscalenären Grube, Processus transversus mit } \\
\text { vorderem und hinterem Tuberkel } \\
\text { C7-Processus transversus nur mit hinterem Tuberkel } \\
\text { C8-keine Tuberkel, auf der ersten Rippe }\end{array}$ & $\begin{array}{l}(\mathrm{C} 5-\mathrm{C} 8) \\
5,66 \pm 1,02 \\
8,98 \pm 1,65 \\
10,43 \pm 1,86 \\
10,76 \pm 2,02 \\
\mathrm{~mm}^{2}\end{array}$ & $\begin{array}{l}\text { Zheng M et al. } 2014 \text {, } \\
\text { Lapegue F et al. } 2014 \text {, } \\
\text { Won SJ et al. } 2012\end{array}$ \\
\hline Plexus brachialis & $\begin{array}{l}\text { Supraclaviculär lateral der A. subclavia über Pleura und erster Rippe, infraclavicu- } \\
\text { lär unter Mm. pectorales } \\
\text { Truncus superior } \\
\text { Truncus medius } \\
\text { Truncus inferior }\end{array}$ & $\begin{array}{l}\mathrm{mm}^{2} \\
16,70 \pm 2,88 \\
14,01 \pm 2,70 \\
13,75 \pm 2,57\end{array}$ & $\begin{array}{l}\text { Zheng M et al. 2014, } \\
\text { Lapegue F et al. } 2014 \text {, } \\
\text { Won SJ et al. } 2012\end{array}$ \\
\hline N. thoracicus longus & $\begin{array}{l}\text { Ursprung aus C5-(C7)/Truncus superior, durchbohrt den M. scalenus medius, lat. } \\
\text { des Plexus, 2. Zacke des M. serratus anterior }\end{array}$ & $1,6 \pm 0,3 \mathrm{~mm}$ & Lieba-Samal D et al. 2014 \\
\hline N. suprascapularis & $\begin{array}{l}\text { Ursprung aus C5/C6/Truncus superior, Verlauf oberfl. auf M. scalenus medius, } \\
\text { lateral des Plexus, unter M. omohyoideus }\end{array}$ & $1,9-2,0 \mathrm{~mm}^{2}$ & Battaglia PJ et al. 2013 \\
\hline N. axillaris & $\begin{array}{l}\text { (A) Arm in Eversions- und Abduktionsstellung: Hiatus axillaris lateralis neben der } \\
\text { A. circumflexia humeri posterior } \\
\text { (B) Dorsaler Zugang: Neurovaskulärer Raum zwischen M. teres minor, M. deltoi- } \\
\text { deus, M. triceps und Humerusschaft: }\end{array}$ & $?$ & $\begin{array}{l}\text { Gruber H et al. 2014, } \\
\text { Rothe C et al. } 2011\end{array}$ \\
\hline N. musculocutaneus & $\begin{array}{l}\text { Durchbohrt den M. coracobrachialis, weiterer Verlauf im M. biceps und brachialis, } \\
\text { sens. Endast: N. cutaneus antebr. lat. }\end{array}$ & $\begin{array}{l}4,2 \pm 2,0 \mathrm{~mm}^{2} \\
\text { (Coracobr.) }\end{array}$ & Tagliafico A et al. 2013 \\
\hline N. radialis & $\begin{array}{l}\text { Axilla - begleitet die A. brachialis profunda } \\
\text { Oberarm - Canalis spiralis des Humerus } \\
\text { Ellenbeuge - zwischen M. brachioradialis und -brachialis } \\
\text { R. Profundus - im M. supinator über Radius } \\
\text { R. superficialis - ab Teilung bis zum Handgelenk verfolgen }\end{array}$ & $\begin{array}{l}7,2 \pm 2,9 \mathrm{~mm}^{2} \\
6,2 \pm 3,1 \mathrm{~mm}^{2} \\
2,3 \pm 1,3 \mathrm{~mm}^{2} \\
2-3 \mathrm{~mm}^{2}\end{array}$ & $\begin{array}{l}\text { Tagliafico A et al. } 2013 \text {, } \\
\text { Marx SC et al. } 2011\end{array}$ \\
\hline N. medianus & $\begin{array}{l}\text { Axilla - zwischen A. axillaris und M. coracobrachialis (ventral) } \\
\text { Oberarm - A. brachialis, ventral im Sulcus bicipitalis medialis } \\
\text { Ellenbeuge - A. brachialis/Teilung, unter M. pronator teres } \\
\text { Unterarm - zwischen oberfl. und tiefen Fingerflexoren } \\
\text { Carpaltunnel - Os lunatum, Os pisiforme }\end{array}$ & $\begin{array}{l}8,9 \pm 1,8 \mathrm{~mm}^{2} \\
5,5 \pm 2,2 \mathrm{~mm}^{2} \\
5,7 \pm 1,3 \mathrm{~mm}^{2} \\
8,2 \pm 2,3 \mathrm{~mm}^{2}\end{array}$ & $\begin{array}{l}\text { Boehm J et al. 2014, } \\
\text { Tagliafico A et al. } 2013\end{array}$ \\
\hline N. ulnaris & $\begin{array}{l}\text { Oberarm - auf medialem Tricepskopf } \\
\text { Sulcus - Epicondylus medialis/Olecranon } \\
\text { Unterarm - A. ulnaris } \\
\text { Handgelenk - Os pisiforme }\end{array}$ & $\begin{array}{l}6,3 \pm 1,7 \mathrm{~mm}^{2} \\
7,6 \pm 1,2 \mathrm{~mm}^{2} \\
5,2 \pm 1,3 \mathrm{~mm}^{2} \\
3,1 \pm 1,0 \mathrm{~mm}^{2}\end{array}$ & $\begin{array}{l}\text { Boehm J et al. 2014, } \\
\text { Tagliafico A et al. } 2013\end{array}$ \\
\hline Fingernerven (volar) & Begleitgefäße oder Tracing vom Hauptstamm des N. ulnaris/medianus & $1,1 \pm 0,01 \mathrm{~mm}$ & Kessler JM et al. 2012 \\
\hline $\begin{array}{l}\text { N. cutaneus ante- } \\
\text { brachii lateralis }\end{array}$ & distale Bicepssehne/Vena cephalica (Oberarm) & $\begin{array}{l}3,3 \mathrm{~mm}^{2} \\
(\mathrm{R}=1,9-5,2)\end{array}$ & Chiavaras MM et al. 2014 \\
\hline $\begin{array}{l}\text { N. cutaneus ante- } \\
\text { brachii medialis }\end{array}$ & Begleitgefäß Vena basilica (Oberarm) & $2,5 \pm 0,4 \mathrm{~mm}$ & Thallaj A. 2011 \\
\hline R. dorsalis n. ulnaris & Abgang aus dem N. ulnaris am Unterarm (neben der A. ulnaris) & $\begin{array}{l}1,6 \mathrm{~mm}^{2} \\
(\mathrm{R}=1,1-2,2)\end{array}$ & Le Corroller T et al. 2013 \\
\hline
\end{tabular}

diagnostische Medizin (AANEM) in einem evidenzbasierten Review unter Berücksichtigung von 45 Publikationen Ende 2012 der Sonografie den Empfehlungsgrad A für die Diagnostik des KTS ausgesprochen [59]. Bezüglich der Korrelation des Schweregrades der Veränderungen im Ultraschall mit den elektrophysiologischen Befunden existieren unterschiedliche Aussagen. Manche Autoren fanden eine positive Korrelation, andere wiederrum nicht $[57,58,69-72]$. Interessanterweise stellte sich auch in den zuletzt publizierten Studien über hereditäre oder erworbene, demyelinisierende Immunneuropathien häufig keinen Zusammenhang zwischen den sonografischen Auffälligkeiten und den klinischen sowie elektrodiagnostischen Befunden heraus
[73-75]. Bis zur Aufklärung dieses Widerspruches sollte aus Sicht des Autors die Sonografie nicht primär zur Diagnostik eines KTS eingesetzt werden. Ausnahmen bilden „stromempfindliche" Patienten und Kinder [3,4]. Andere Untersuchungsansätze (z.B. Bestimmung der palmaren Wölbung des Retinaculum flexorum oder die Quantifizierung der hypoechogenen Veränderungen der Faszikel) haben sich bisher nicht durchsetzen können [76]. Das trifft auch auf die häufig gesteigerte epi- und perineurale Vaskularisation proximal der Kompressionsstelle zu. In einem 2014 publizierten Review bemängelten die Autoren die bisher fehlende Standardisierung der Farboppleruntersuchung sowie das Studiendesign mancher Arbeiten. Sie fanden eine 
Tab. 1b Nerven, anatomische Landmarken und Normwerte des CSA $\left(\mathrm{mm}^{2}\right)$ bzw. des Anterior-Posterioren Durchmessers (mm) \pm Standardabweichung Bauchwand und untere Extremität. ( $R=$ Range), ${ }^{*}$ Mittelwert/Standardfehler, [15-40].

\begin{tabular}{|c|c|c|c|}
\hline Nerv & Landmarke & Normwerte & Referenz \\
\hline $\begin{array}{l}\text { N. iliohypogastricus } \\
\text { N. ilioinguinalis } \\
\text { N. genitofemoralis }\end{array}$ & $\begin{array}{l}\text { Sonde senkrecht zum Leistenkanal sowie kranial und hinter der Spina iliaca } \\
\text { anterior superior - 3-eck zwischen Beckenkamm sowie M. obliquus internus } \\
\text { abdominus und M. transversus abdominis, Begleitgefäß A. circumflexa ilium } \\
\text { profunda } \\
\text { Ramus genitalis n. genitofem. im Leistenkanal }\end{array}$ & $?$ & Soneji N et al. 2013 \\
\hline $\begin{array}{l}\text { N. cutaneus femoralis } \\
\text { lateralis }\end{array}$ & Intermuskulärer Raum zwischen M. tensor fasciae latae und M. sartorius & $1,04 \pm 0,44 \mathrm{~mm}^{2}$ & Zhu J et al. 2012 \\
\hline N. femoralis & $\begin{array}{l}\text { In Höhe Leistenband lateral der A. femoralis zwischen den Blättern der Faszia } \\
\text { iliaca auf dem M. iliacus }\end{array}$ & $* 15,2 / 2 \mathrm{~mm}^{2}$ & Tagliafico A et al. 2012 \\
\hline N. obturatorius & $\begin{array}{l}\text { - In Höhe Leistenband medial der V. femoralis/M. pectineus } \\
\text { - vorderer Ast zwischen M. adductor longus und -brevis } \\
\text { - hinterer Ast zwischen M. adductor brevis und -magnus } \\
\text { - Hauptast zwischen M. pectineus und M. adductor long. }\end{array}$ & $\begin{array}{l}\text { AP Diameter } \\
1,4 \pm 0,6 \mathrm{~mm} \\
1,7 \pm 0,6 \mathrm{~mm} \\
2,7 \pm 1,2 \mathrm{~mm}\end{array}$ & $\begin{array}{l}\text { Soong J et al. 2007, } \\
\text { Simeoforidou M et al. } 2013\end{array}$ \\
\hline N. saphenus & $\begin{array}{l}\text { Distaler Oberschenkel - zwischen M. sartorius und M. vastus medialis, } \\
\text { Begleitgefäß A. femoralis } \\
\text { Knie/Unterschenkel - Vena saphena magna }\end{array}$ & $* 1,5 / 6 \mathrm{~mm}^{2}$ & $\begin{array}{l}\text { Saranteas T et al. 2011, } \\
\text { Tagliafico A et al. } 2012\end{array}$ \\
\hline N. ischiadicus & $\begin{array}{l}\text { Proximal - unter M. gluteus maximus und M. piriformis } \\
\text { Distal - Fossa poplitea (Bifurkation in N. tibialis und fibularis) }\end{array}$ & $\begin{array}{l}* 41,2 / 15 \mathrm{~mm}^{2} \\
{ }^{*} 33,1 / 7 \mathrm{~mm}^{2}\end{array}$ & Tagliafico A et al. 2012 \\
\hline N. tibialis & $\begin{array}{l}\text { Proximal - Fossa poplitea/Bifurkation N. ischiadicus/A. tibialis } \\
\text { Distal - Tarsaltunnel, A. tibialis posterior }\end{array}$ & $\begin{array}{l}* 9,9 / 6 \mathrm{~mm}^{2} \\
* 9,6 / 4 \mathrm{~mm}^{2}\end{array}$ & Tagliafico A et al. 2012 \\
\hline N. suralis & $\begin{array}{l}\text { Prox. - Fossa poplitea, Verlauf zwischen den Köpfen des Gastrocn. } \\
\text { Distal - lateral Archillessehne, Vena saphena parva }\end{array}$ & $\begin{array}{l}1,8 \pm 0,6 \\
\mathrm{~mm}^{2}\end{array}$ & Boehm et al. 2014 \\
\hline N. peroneus communis & $\begin{array}{l}\text { Ischiadicusbifurkation, unter Caput breve des M. biceps femoris, } \\
\text { Fibulakopf }\end{array}$ & $\begin{array}{l}8,9 \pm 2,0 \mathrm{~mm}^{2} \\
* 13,2 / 14 \mathrm{~mm}^{2}\end{array}$ & $\begin{array}{l}\text { Boehm et al. 2014, } \\
\text { Tagliafico A et al. } 2012\end{array}$ \\
\hline $\begin{array}{l}\text { N. peronaeus } \\
\text { superficialis }\end{array}$ & $\begin{array}{l}\text { Distaler Unterschenkel - unter der Fascia cruris zwischen M. peroneus brevis } \\
\text { und M. extensor digitorum longus }\end{array}$ & $?$ & Chin KJ 2013 \\
\hline
\end{tabular}

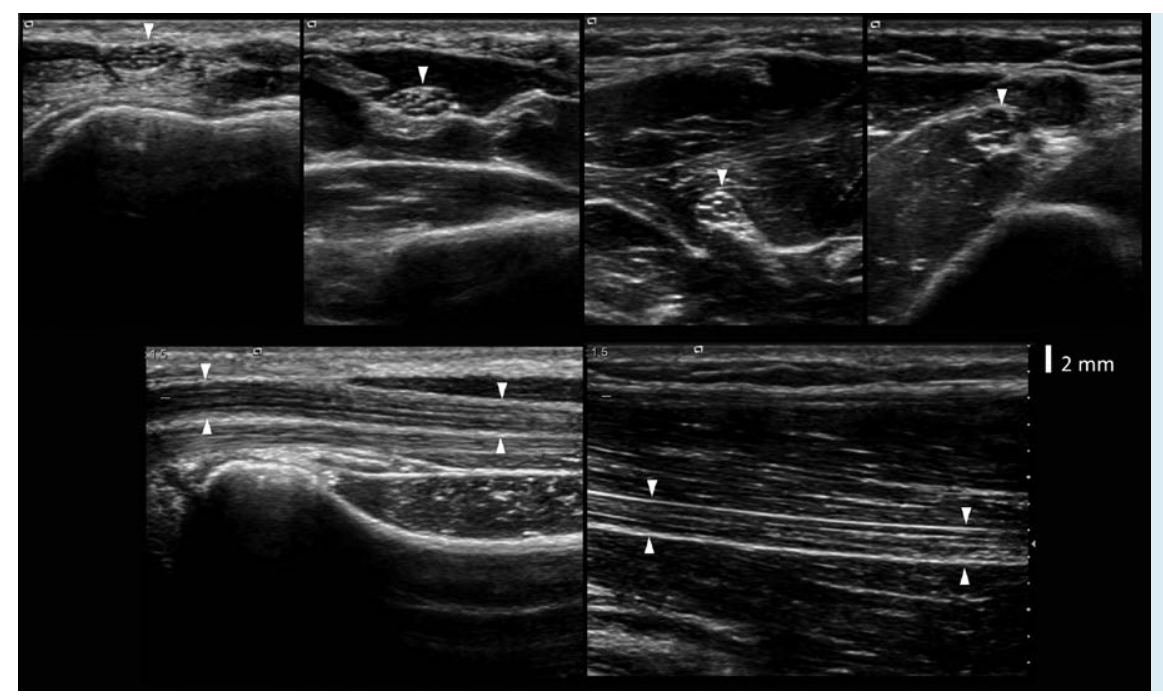

Abb. 1 Normale Sonoanatomie des peripheren Nervs. Oben axiale Scans des N. medianus (Pfeilköpfe) von der Axilla (rechts) bis zum Karpaltunnel (links). Beachte die zunehmende Anzahl der Faszikel von proximal nach distal. Unten Längsschnitte in Höhe Karpaltunnel (links) und am Unterarm rechts. Pfeilköpfe N. medianus.

\section{Autor}

Boehm J et al. 2014

Burg EW et al. 2014

Tagliafico A et al. 2013

Zaidman CM et al. 2009

Burg EW et al. 2014

Wanitwattanarumlug B et al. 2012

Kim MK et al. 2014

Azami A et al. 2014

CSA N. medianus
$\mathrm{MW} \pm \mathrm{SD}$
$\left(\mathrm{mm}^{2}\right)$

$8,5+1,8$

$8,3 \pm 1,9$

$8,2 \pm 2,3$

$9,7 \pm 1,9$

$7,0 \pm 1,1$

$6,8 \pm 0,9$

$7,9 \pm 1,3$

$8,5 \pm 0,8$

CSA N. medianus
Oberer Grenzwert
$\left(\mathrm{mm}^{2}\right)$

10,3

10,2

10,5

11,6

8,1

7,7

9,2

9,3

\section{Population}

Europa (Deutschland, Ungarn)

Europa (Niederlande)

Europa (Italien)

Nordamerika (USA)

Asien (Indien)

Asien (Thailand)

Asien (Korea)

Asien (Iran)
Tab. 2 CSA am Karpaltunneleingang und geografische Herkunft [27, 29, 53, 55-58].

CSA = Nervenquerschnittsfläche; $M W=$ Mittelwert; SD = Standartabweichung 


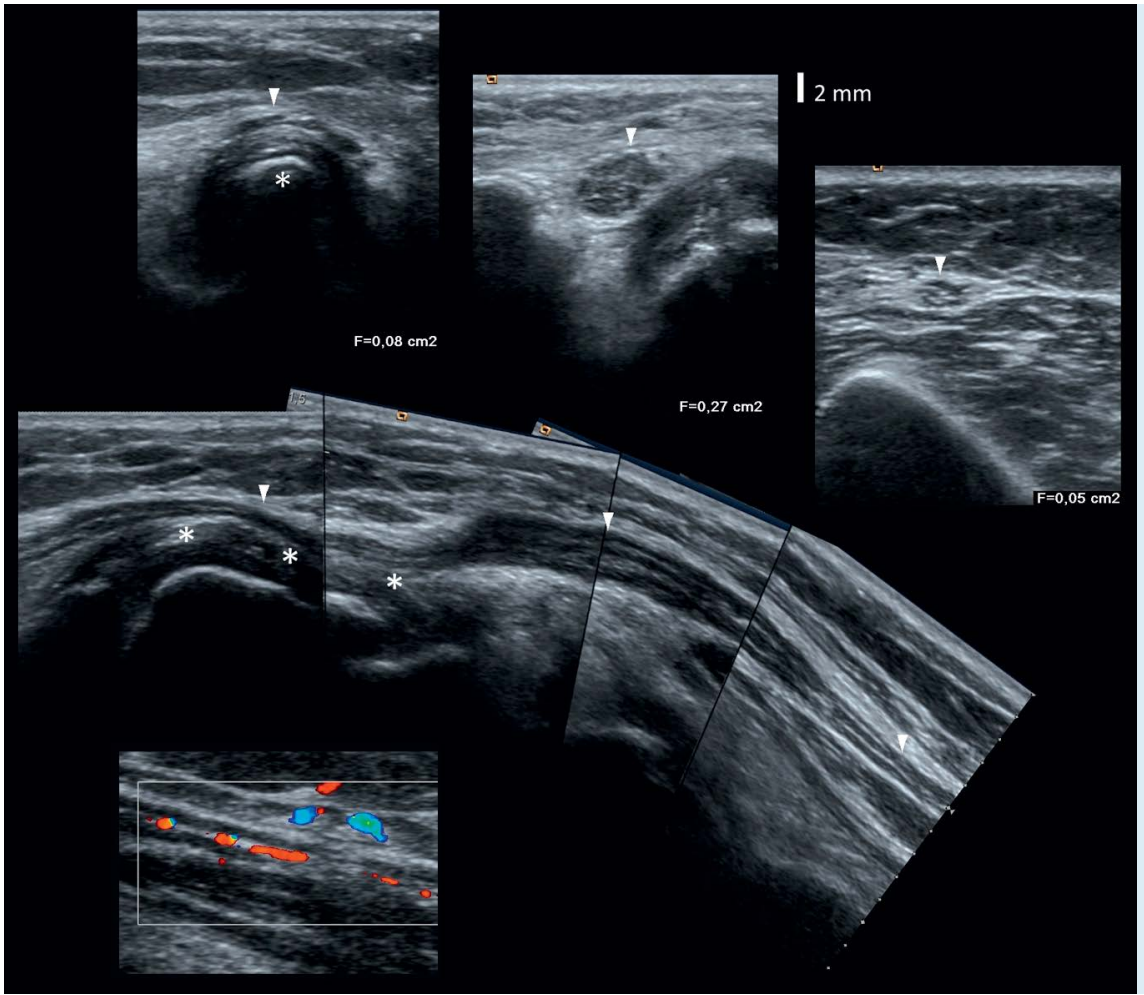

Abb. 2 Sonografische Charakteristika eines Engpasssyndroms (hier Kubitaltunnelsyndrom). Oben axiale Scans von proximal (rechts) nach distal (links) korrelierend mit der Panoramarekonstruktion (Mitte). Pfeilköpfe N. ulnaris. Sterne Kompression zwischen Kubitaltunnelretinakulum und Humeroulnargelenk. Beachte die Abflachung an der Kompressionsstelle und das Ödem proximal davon mit Verlust der normalen Echotextur sowie pathologischer Zunahme der Nervenquerschnittsfläche. Unten: epineurale Hypervaskularisation proximal der Kompressionsstelle.

\begin{tabular}{|c|c|c|c|}
\hline \multicolumn{4}{|l|}{ Karpaltunnelsyndrom } \\
\hline $\mathrm{CSA}_{\text {Handgelenk }}($ Cut-Off $) \geq 8,5 \mathrm{~mm}^{2}$ & $\begin{array}{l}\text { Sensitivität 97\%, } \\
\text { Spezifität 98\% }\end{array}$ & $\begin{array}{l}\text { Iran, } \\
\text { prospektiv }\end{array}$ & Mohammadi A et al. 2010 \\
\hline $\mathrm{CSA}_{\text {Handgelenk }}($ Cut-Off $) \geq 10 \mathrm{~mm}^{2}$ & $\begin{array}{l}\text { Sensitivität } 82 \% \text {, } \\
\text { Spezifität } 87 \%\end{array}$ & $\begin{array}{l}\text { Schweiz, } \\
\text { prospektiv }\end{array}$ & Ziswiler HR et al. 2005 \\
\hline WFR $_{\text {Handgelenk/Unterarm }} \geq 1,4$ & Sensitivität $100 \%$ & $\begin{array}{l}\text { USA, } \\
\text { prospektiv }\end{array}$ & Hobbson-Web LD et al. 2008 \\
\hline $\mathrm{CSA}_{\text {Handgelenk }}($ Cut-Off $) \geq 9 \mathrm{~mm}^{2}$ & $\begin{array}{l}\text { Sensitivität } 87 \% \text {, } \\
\text { Spezifität } 83 \%\end{array}$ & Metaanalyse & Tai TW et al. 2012 \\
\hline \multicolumn{4}{|l|}{ Kubitaltunnelsyndrom } \\
\hline $\mathrm{CSA}_{\text {MaxSulcus }}($ Cut-Off $) \geq 10 \mathrm{~mm}^{2}$ & $\begin{array}{l}\text { Sensitivität } 88 \% \text {, } \\
\text { Spezifität } 88 \%\end{array}$ & $\begin{array}{l}\text { Italien, } \\
\text { prospektiv }\end{array}$ & Volpe A et al. 2009 \\
\hline $\mathrm{HCR}_{\text {Sulcus/Oberarm }} \geq 1,4$ & $\begin{array}{l}\text { Sensitivität } 86 \% \text {, } \\
\text { Spezifität } 87 \%\end{array}$ & $\begin{array}{l}\text { Österreich, } \\
\text { prospektiv }\end{array}$ & Gruber $\mathrm{H}$ et al. 2009 \\
\hline \multicolumn{4}{|c|}{ Peroneusneuropathie am Fibulakopf } \\
\hline $\mathrm{CSA}_{\text {Fibulakopf }}($ Cut-Off $) \geq 9 \mathrm{~mm}^{2}$ & $\begin{array}{l}\text { Sensitivität 90\%, } \\
\text { Spezifität 69\% }\end{array}$ & $\begin{array}{l}\text { Niederlande, } \\
\text { retrospektiv }\end{array}$ & Visser LH et al. 2013 \\
\hline \multicolumn{4}{|c|}{ Meralgie (Kompression des N. cutaneus femoris lateralis) } \\
\hline $\operatorname{CSA}_{\text {Spina }}($ Cut-Off $) \geq 5 \mathrm{~mm}^{2}$ & $?$ & $\begin{array}{l}\text { Korea, } \\
\text { retrospektiv }\end{array}$ & Suh DH et al. 2013 \\
\hline \multicolumn{4}{|c|}{ Supinatortunnelsyndrom (Kompression des Ramus profundus $\mathbf{n}$. radialis) } \\
\hline AP-Durchmesser $r_{\text {Eingang }} \geq 1,5 \mathrm{~mm}$ & $?$ & $\begin{array}{l}\text { Österreich, } \\
\text { retrospektiv }\end{array}$ & Djurdjevic T et al. 2013 \\
\hline
\end{tabular}

Tab. 3 Ausgewählte Diagnoseparamater für Engpasssyndrome, $[44,45,61-68]$. durchschnittliche Sensitivität von 72\% (Range 41-95\%) sowie eine Spezifität von $88 \%$ (Range 71-100\%) [77]. Weitere interessante Ansätze sind die Quantifizierung der verminderten Deformierbarkeit und Mobilität des N. medianus bei KTS-Patienten, wenn die Finger gebeugt werden [78] sowie Steifigkeitsmessungen des N. medianus mittels Scherwellenelastografie [6]. Die zuletzt genannte Methode zeigte in der angegeben Studie eine Korrelation mit dem Schweregrad des KTS sowie eine Sensitivität und Spezifität von 93.3 vs. 88.9\% [6]. Das Kubitaltunnelsyndrom (KUTS) wurde bisher in deutlich weniger Arbeiten unter- sucht und es existieren zurzeit keine evidenzbasierten Empfehlungen. Einen Überblick gibt $\bullet$ Tab. 3 [45,64]. Neue, dynamische Untersuchungen zeigen in Abhängigkeit vom Grad der Beugung im Ellenbogengelenk zwischen 90 und $120^{\circ}$ eine Verlagerung des N. ulnaris nach ventral (medial) sowie dessen zunehmende Abflachung im Kubitaltunnel und auch im Sulcus, wenn dieser flach angelegt ist. Eine leichte Beugung zwischen 30 und $90^{\circ}$ beeinflusst hingegen die Messung der Nervenquerschnittsfläche nicht signifikant $[79,80]$. Bei $32 \%$ der Patienten fand sich darüber hinaus eine Luxation des N. ulnaris über den Epicondylus 
medialis bei Flexion im Ellenbogengelenk [81]. Diese Noxe führt wahrscheinlich zu einer reaktiven Fibrose des Epineuriums, was sich sonografisch in einer Verdickung des hyperechogenen Epineuriums äußern kann [82]. „Cut-Off“ Werte für die Diagnostik seltenerer Nervenkompressionssyndrome sind nur beschränkt verfügbar. Es gibt derzeit nur wenige retrospektive Studien. In $\odot$ Tab. 3 finden sich Angaben für die Meralgie, für das Supinatorlogensyndrom und für die Neuropathie des N. peroneus communis am Fibulakopf [66-68]. Bei anderen Krankheitsbildern muss man sich an den publizierten Normwerten der Nervenquerschnittsfläche unter Berücksichtigung der demografischen Abhängigkeiten orientieren. Neben der Verwendung des Ultraschalls zur apparativen Diagnostik eines Engpasssyndromes liefert er 2 Zusatzinformationen, die mit der Elektrophysiologie allein nicht gewonnen werden können [3,4]. In die erste Kategorie fallen anatomische Varianten wie z.B. die hohe Teilung des N. medianus, akzessorische Gefäße (A. mediana) und Muskeln oder atypisch abgehende Äste. Die amerikanische Vereinigung für neuromuskuläre und elektrodiagnostische Medizin (AANEM) hat in o.g. Review der Sonografie den Empfehlungsgrad B zur Detektion solcher Anomalien ausgesprochen [59]. Sie können bei endoskopischen Operationstechniken in der Lernphase des Operateurs ggf. Anlass für intraoperative Komplikationen (Nervenverletzungen, Blutungen) sein [83]. Darüber hinaus fand sich in früheren Arbeiten ein höherer Anteil solcher Varianten bei symptomatischen Patienten mit einem KTS, verglichen mit der asymptomatischen Normalbevölkerung [84]. Neuere prospektive Studien an größeren Populationen zeigen jedoch, dass die hohe Teilung des $\mathrm{N}$. medianus mit und ohne A. mediana nicht als unabhängiger Risikofaktor für die Entwicklung eines KTS zu betrachten ist $[85,86]$. Die Inzidenz einer A. mediana betrug bei lateinamerikanischen Geflügelverarbeitern 3,7\% und die eines bifiden N. medianus 8,6\% [85]. Auch verlängerte Muskelbäuche der oberflächlichen Fingerflexoren oder akzessorische Mm. lumbricales stellen bei asymptomatischen Individuen keinen Risikofaktor für die Entwicklung eines KTS dar (Untersuchungszeitraum 1 Jahr), während bei symptomatischen Patienten eine Assoziation mit dem Krankheitsbild beobachtet wurde [87]. In die zweite Kategorie fallen die genaue Aufklärung der Ursache eines Kompressionssyndroms und die Ermittlung mehrerer Kompressionsstellen. Beides beeinflusst gegebenenfalls das weitere therapeutische Vorgehen. Bei allen Engpasssyndromen lässt sich die Kompression durch Bänder, Tenosynovitiden, Knochen, akzessorische Muskeln, Raumforderungen wie Ganglienzysten sowie andere Neoplasien neuralen und nichtneuralen Ursprungs sonografisch darstellen [87-92]. Besonders beim Kubitaltunnelsyndrom existieren manchmal mehrere Kompressionsstellen oder eine atypisch lange Kompression bei degenerativen Veränderungen des Sulcus/Kubitaltunnels nach vorausgegangener Ellenbogenfraktur (Ulnarisspätparese), die sonografisch detektiert werden können [90]. Beispiele seltener Nervenkompressionssyndrome und sekundärer Ursachen zeigt $\bullet$ Abb. 3. Wenn auch keine Korrelation der präoperativ bestimmten Nervenquerschnittsfläche mit dem Outcome nach einer KTS-Operation besteht [93], so ermöglicht die Sonografie den Nachweis einer fokalen Reststenose nach erfolgloser chirurgischer Intervention durch Narbengewebe oder Reste des Retinaculum flexorum [94,95]. Nach der Erfahrung des Autors stellt der Nachweis von Reststenosen als Ursache persistierender Beschwerden nach chirurgischer Intervention bei den anderen Kompressionssyndromen

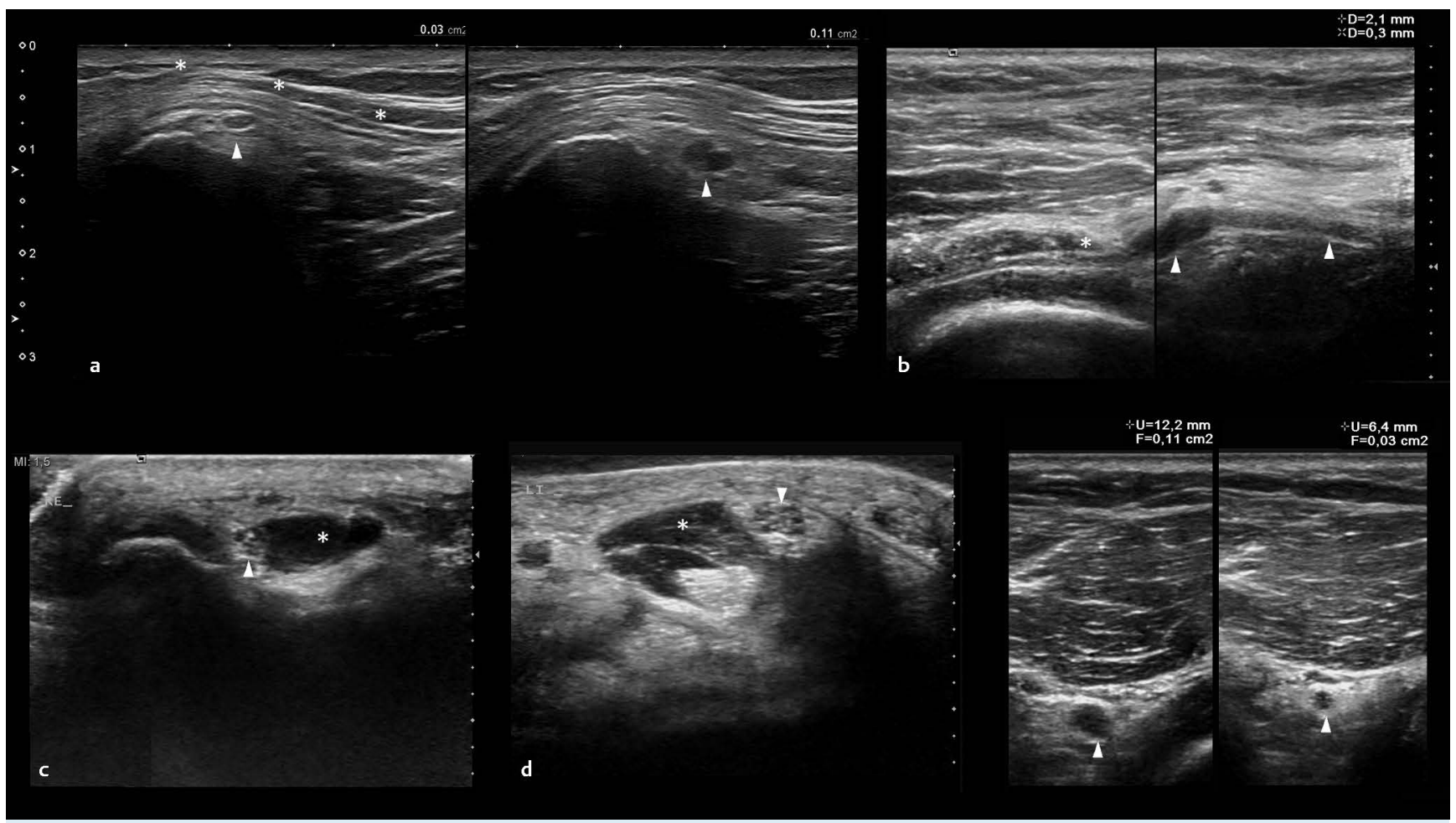

Abb. 3 Seltene Kompressionssyndrome und sekundäre Ursachen. a Kompression des N. cutaneus femoris lateralis (Pfeilkopf) zwischen Spina iliaca anterior superior und Leistenband (Sterne). Im rechten Bildteil ist das Ödem proximal der Kompressionsstelle gut zu erkennen. b Supinatortunnelsyndrom im longitudinalem Scan (oben) von proximal (rechts) nach distal (links) und korrespondierenden axialen Scans (unten). Auch hier ist das fokale Ödem des Ramus profundus n. radialis (Pfeilköpfe) proximal der Kompressionsstelle beim Eintritt in den M. supinator (Stern) gut zu sehen. c Irritation des N. ulnaris (Pfeilkopf) durch ein Ganglion (Stern) in der Loge de Guyon. d Verlängerte Muskelbäuche (Stern) bedrängen den N. medianus (Pfeilkopf) beim Eintritt in den Karpaltunnel. 


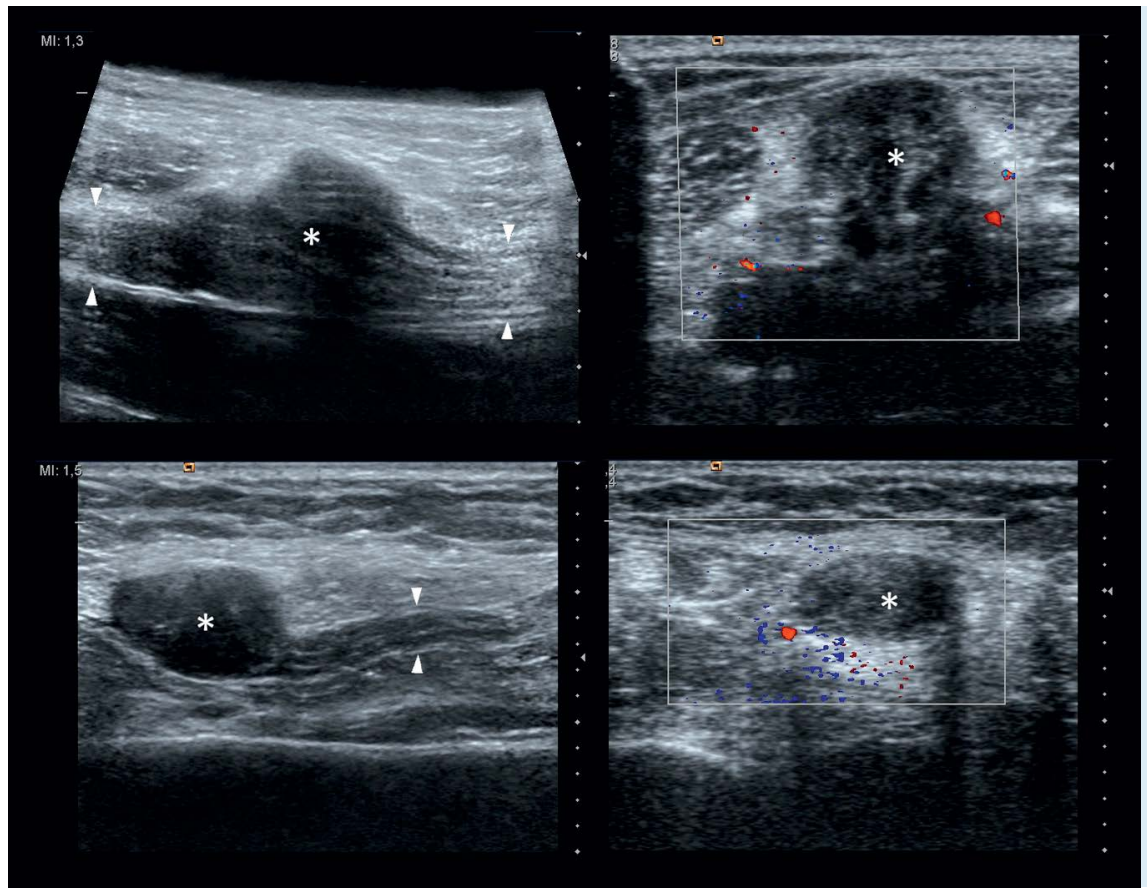

Abb. 4 Hochgradige traumatische Nervenverletzungen (Sunderland IV-V). Oben nicht vaskularisiertes Kontinuitätsneurom (Sterne) des N. tibialis (Pfeilköpfe) nach Pistolenschussverletzung. Unten: Nicht vaskularisiertes Stumpfneurom (Sterne) des N. ulnaris (Pfeilköpfe) am Oberarm nach Kontinuitätsdurchtrennung.

eine weitere Anwendungsmöglichkeit der Methode dar. Die Sonografie kann außerdem zur ultraschall-gestützten Schmerztherapie besonders bei Patienten mit Meralgie eingesetzt werden [96].

\section{Anwendungsmöglichkeiten bei traumatischen Nervenverletzungen}

Nach einer klinisch kompletten Nervenläsion nach geschlossenem Trauma stellt sich immer die Frage, ob der natürliche Verlauf der Reinnervation unter konservativer Therapie abgewartet werden kann, oder ob eine primäre oder sekundäre Nervenrekonstruktion zur Wiederherstellung der Funktion unumgänglich ist. Dabei spielt der Faktor Zeit eine wesentliche Rolle. Je mehr davon ohne erfolgreiche Reinnervation vergeht, desto größer ist die Wahrscheinlichkeit, dass sich irreversible Schäden am Muskel oder am peripheren Nerven selbst einstellen [97-99]. Orientierend an der SUNDERLAND Klassifikation betrifft das vor allem die Grade IV und V, die entweder eine großflächige Zerstörung des Perineuriums oder einen kompletten Verlust der Nervenkontinuität zur Folge haben $[99,100]$. Durch den irreversiblen Ersatz des Endorgans Muskel durch Bindegewebe und/oder das reiinnervationsbehindernde Einwachsen von Bindegewebe in die Faszikel und das Nervenhüllgewebe (perineurale Fibrose) verschlechtert sich naturgemäß die Prognose für den Patienten [97-99]. Die elektromyografische Untersuchung gestattet zwar die Frage nach einer ablaufenden Reinnervation zu beantworten. Hierfür müssen allerdings bis zu 3 Monate abgewartet werden [101]. Verwendet man dagegen die Sonografie kann diese Problematik mit hoher Genauigkeit (93,2\%) zu einem früheren Zeitpunkt geklärt werden [102-104]. Eine Grad-V-Läsion ist praktisch sofort durch die fehlende Nervenkontinuität, spätestens jedoch nach Bildung eines Neuroms am proximalen Stumpf nachweisbar. Eine Grad-IV-Läsion lässt sich im Verlauf durch die Entstehung eines Kontinuitätsneuroms detektieren. Solche Neurome erinnern sonografisch an eine Kaulquappe (Stumpfneurom) oder zeigen eine spindelförmige Auftreibung des Nervs im Längsschnitt (Kontinuitätsneurom). Sie sind meist nicht vaskularisiert sowie homogen bis inhomogen hypoechogen [102106]. Die $\diamond$ Abb. 4 illustriert 2 Beispiele. Darüber hinaus ist die Sonografie durch die Möglichkeit der millimetergenauen Lokalisation einer Läsion sowie durch den Nachweis von Mehretagenverletzungen der Elektrophysiologie überlegen. Dies kann z.B. für das präoperative Mapping (kutan oder Drahtmarkierung) vorteilhaft verwendet werden [107]. Die oben gemachten Ausführungen treffen auch auf den Plexus brachialis zu. So berichten einige Autoren über eine Sichtbarkeitsrate der Rami ventrales C5-C7 von $100 \%$, C8 von $92 \%$ und Th1 von $51 \%$. Dabei konnten alle Wurzelaus- und Abrisse detektiert werden (ausgenommen 2 Th1-Ausrisse) [20]. Nach der Erfahrung des Autors sind Wurzelabrisse durch eine Kontinuitätsunterbrechung und Wurzelausrisse durch eine mehr oder weniger große Meningozele im Foramen des Processus transversus charakterisiert. Zu ähnlichen Ergebnissen gelangen auch andere Autoren [108]. Selbst bei Neugeborenen lassen sich die Nervenwurzeln C5-C8 gut darstellen [109]. All diese Ergebnisse haben dazu geführt, dass führende Neurochirurgen Deutschlands bei geschlossenen Traumen und fehlender klinischer Regeneration die Sonografie neben der 3-Tesla-MR-Neurografie als primäres Untersuchungsverfahren empfehlen [97]. Dabei kann die Sonografie neben der Neurografie auch intraoperativ und bed-side angewendet werden [110]. Ähnlich wie bei den Engpasssyndromen bietet die Methode außerdem den Vorteil, dass sich häufig die Ursache einer Nervenläsion (Kallus, Knochenfragmente, Osteosynthesematerial, Hämatom, Narbengewebe usf.) ermitteln lässt [103, $106,111,112]$.

\section{Anwendungsmöglichkeiten bei Raumforderungen peripherer Nerven}

Benigne solitäre Nervenscheidentumore (Neurinom, Neurofibrom, Granularzelltumor, Hybridtumore) [113] sind insgesamt selten und sonografisch unter anderem dadurch charakterisiert, dass die verbliebenen gesunden Faszikel eines peripheren 


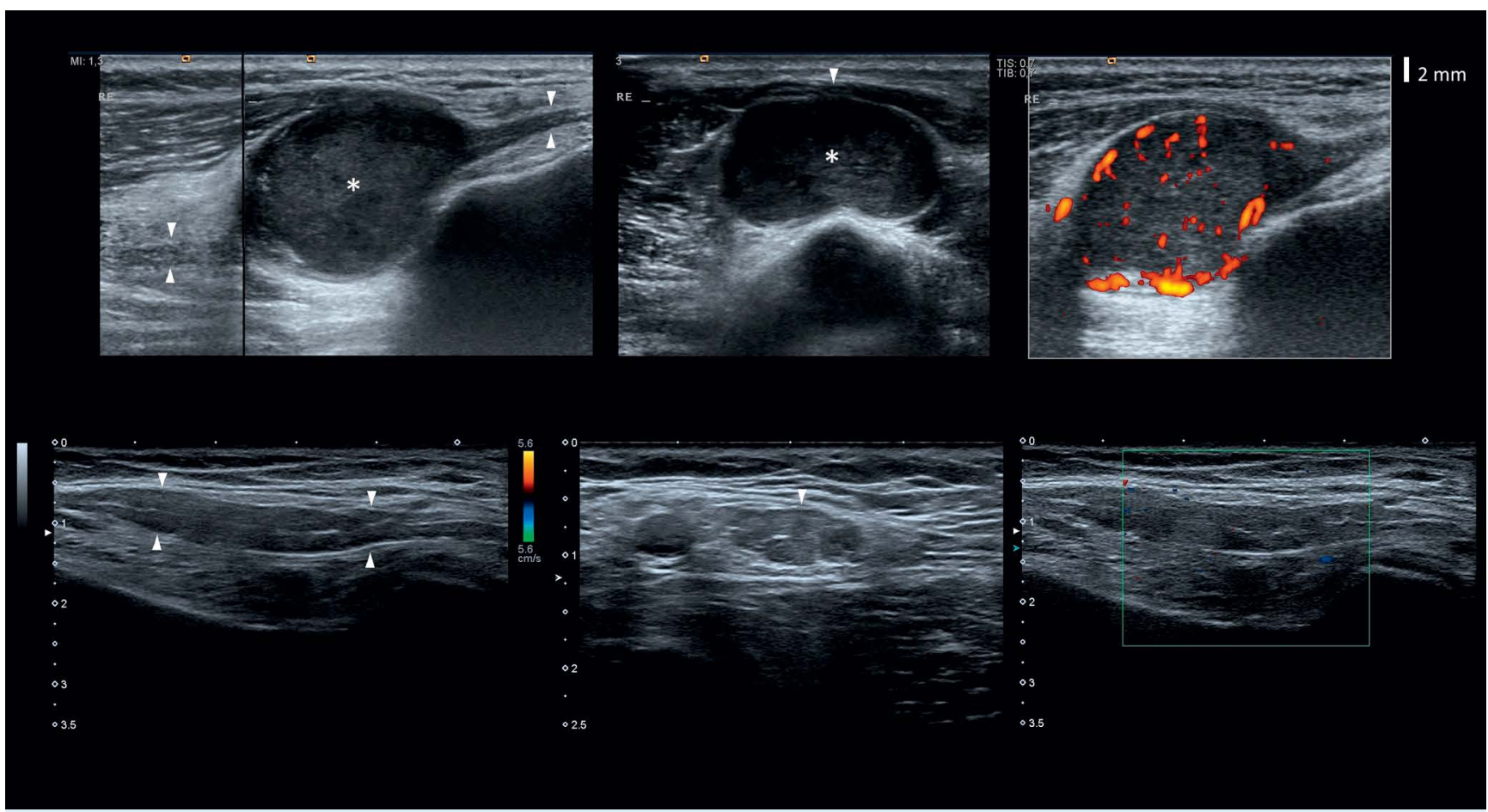

Abb. 5 Solitäre und plexiforme Nervenscheidentumore. Oben Schwannom oder Neurinom (Sterne) des N. peroneus communis (Pfeilköpfe) am Fibulakopf. Beachte den Ein- und Austritt des peripheren Nervs sowie die solitäre, homogen-hypoechogene gut vaskularisierte Tumormatrix. Unten plexiform wachsender Nervenscheidentumor des N. ulnaris (Pfeilköpfe) in der Axilla mit fusiformer Auftreibung und Zerstörung der normalen Echotextur ohne nennenswerte Vaskularisation.

Nervenstammes oder einer Nervenwurzel über der Tumormatrix eintreten und sie auf diese Weise auch wieder verlassen (Spezifität: 86\%, Sensitivität: 63\%) [114,115]. Klinisch zeichnen sie sich durch ein sehr langsames Wachstum über viele Jahre hinweg aus. Neurologische Ausfälle sowie Auffälligkeiten in der Elektrodiagnostik sind selten [116,117]. Ihre Form (rundlich, ovoid usw.) und zahlreiche andere morphologische Merkmale tragen allerdings nicht zu einer Artdiagnose bei [118]. Die Tumormatrix der Neurinome geht häufig von einem funktionslosen Faszikel aus, während in ein Neurofibrom mehrere Faszikel einstrahlen können $[116,117]$. Sie ist bei kleinen Tumoren meist homogen-hypoechogen und kann im gealterten Stadium regressive Veränderungen in Form einer inhomogenen Echotextur sowie Zystenbildungen aufweisen [119]. ๑ Abb. 5 zeigt als Beispiel ein Schwannom. Mit zunehmender Größe ist zudem die gut ausgeprägte und hierarchisch gegliederte Gefäßarchitektur mittels Farbdoppler darstellbar [120]. Solche Raumforderungen sind meist scharf von der Umgebung abgegrenzt mit Ausnahme des Granularzelltumors [119]. Informationen über Position und Lokalisation der gesunden Faszikel und des Tumors sind für den Neurochirurgen präoperativ von entscheidender Bedeutung. In einer unlängst veröffentlichten Arbeit wurde gezeigt, dass sich sonografisch die normalen Faszikel in guter Übereinstimmung mit der MR-Traktografie darstellen lassen [121]. Besteht der Verdacht auf einen peripheren Nervenscheidentumor sollte dieser unter mikrochirurgischen Kautelen entfernt werden. Eine Biopsie birgt die Gefahr der Verletzung gesunder Faszikel $[116,117]$. Elastografisch sind Schwannome steifer als das umgebende Gewebe [5]. Bei der Schwannomatose, Neurofibromatose Typ I und Segmentneurofibromatose können multiple solitäre Raumforderungen entlang der Nervenstämme und Wurzeln vorkommen [119]. Solitäre maligne Nervenscheidentumore treten noch viel seltener auf und lassen sich pathohistologisch in diverse
Entitäten unterteilen [113]. Ein wichtiges klinisches Malignitätskriterium stellt die rasche Größenzunahme der Raumforderung mit frühzeitig auftretenden neurologischen Ausfällen dar [116,117]. Sonografische Charakteristika (infiltratives Wachstum, irreguläre Tumoroberfläche, regressive Veränderungen der Tumormatrix) weisen nur eine geringe Spezifität (38\%) auf, da solche Veränderungen auch bei benignen Raumforderungen (z.B. Neurinom) beobachtet werden können [120]. In der bislang einzigen Studie, wo die kontrastunterstützte Sonografie (CEUS) bei diversen Weichteilraunforderungen zum Einsatz kam, berichten die Autoren über einen positiven prädiktiven Wert (PPV) von $86 \%$ hinsichtlich der Aussage über die Malignität eines solchen Tumors wenn Perfusionsmuster, Tumorgröße und Lokalisation berücksichtigt werden [122]. Der generelle Einsatz dieser Methode ist aber gerade bei peripheren Nervenscheidentumoren fragwürdig, da besonders gealterte Neurinome ein ebenfalls malignitätsverdächtiges (inhomogenes) Perfusionsmuster aufweisen können. Ein weiterer Ansatz ergibt sich bei größeren und gut vaskularisierten Raumforderungen in der Analyse des Gefäßmusters. Kriterien wie eine anarchisch angelegte Gefäßarchitektur, Stenosen, Verschlüsse und andere Auffälligkeiten sprechen im Falle von 2 positiven Kriterien mit einer Sensitivität und Spezifität von 94 bzw. 93\% für einen malignen Tumor [120]. Einzelne kasuistische Beiträge berichten darüber hinaus über solitäre Raumforderungen im peripheren Nerven nichtneuralen, sondern mesenchymalen Ursprungs. Beispiele für diese Raritäten sind intraneurale Angiome und Lipome, die ebenfalls sonografisch lokalisiert werden können [123-126]. Eine Ausnahme bildet die Gruppe von Neoplasien mit netzförmigem (plexiformen) Wachstum ( $\bullet$ Abb. 5). Wesentliche Vertreter sind das plexiforme Neurofibrom und das Perineuriom [116,117]. Obwohl es sich hier meist um benigne Entitäten handelt, stellen sich nach längerem Verlauf durch das faszikelzerstörende 


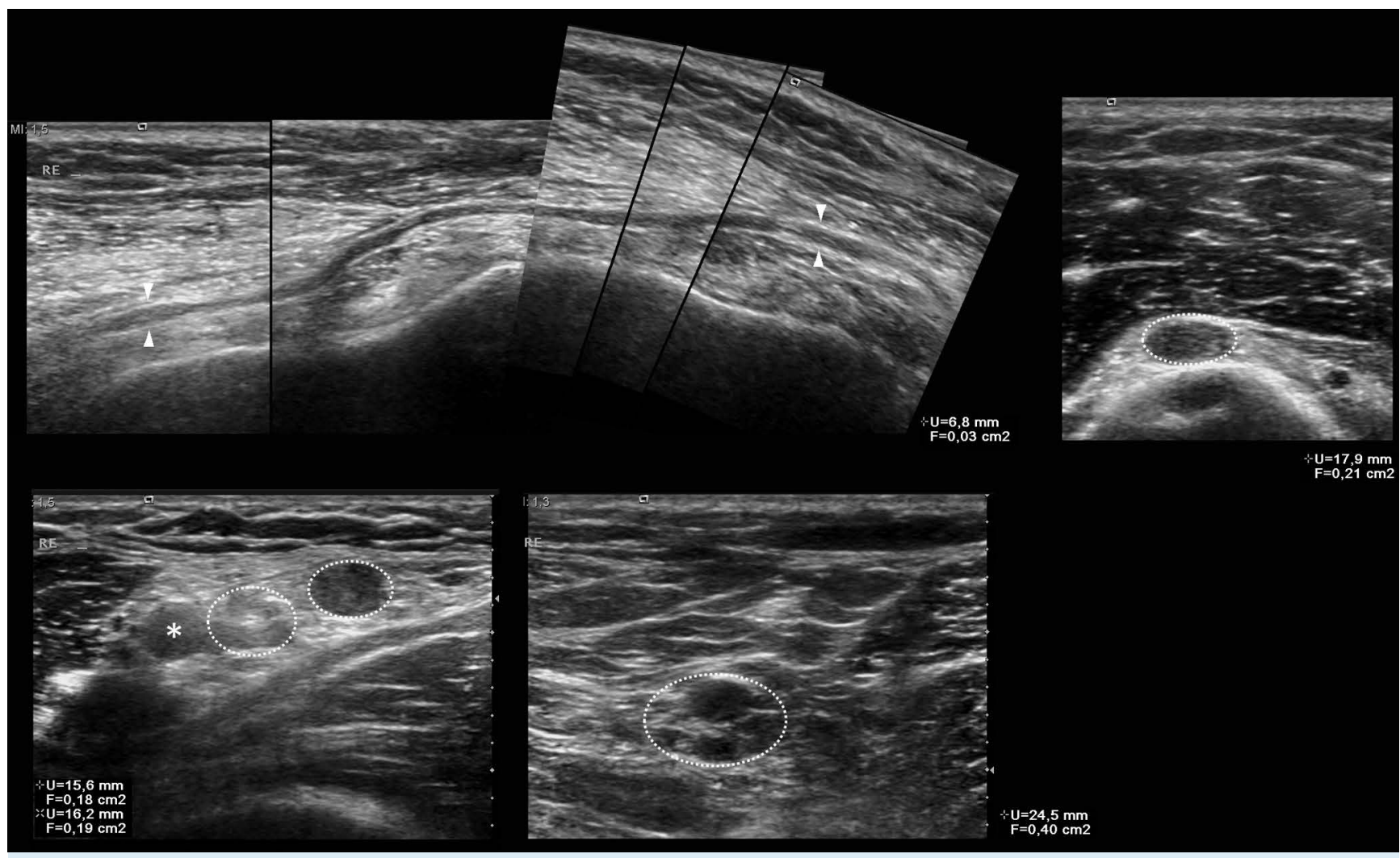

Abb. 6 Fokale Variante einer Immunneuropathie. Klinisch war der Patient zunächst durch „Fallfinger“ rechts auffällig. Die Dekompression des Ramus profundus im Supinatortunnel brachte keine Befundbesserung. Oberer Bildteil links: Ramus profundus n. radialis (Pfeilköpfe) postoperativ im Supinatortunnel. Eine pathologische Veränderung ist nicht zu erkennen (vergl. auch mit Abb. 3b). Dagegen zeigen der rechte N. radialis am Oberarm (oberer
Bildteil rechts) und in der Axilla (unterer Bildteil links) eine Zerstörung der normalen Echotextur mit Vergrößerung der Nervenquerschnittfläche. Dies trifft auch auf den rechten N. ulnaris in der Axilla zu (unterer Bildteil links). Beide Nerven sind größer als die A. axillaris (Stern). Eine weitere fokale Hypertrophie zeigt der rechte N. tibialis in der Kniekehle (unterer Bildteil rechts). Alle anderen Nerven waren sonografisch nicht betroffen.
Wachstum meist irreversible axonale Schäden ein. Nach der Erfahrung des Autors (bisher sonografischer Nachweis von 10 solchen Raritäten mit histologischer Sicherung) sind sie sonografisch durch eine hypoechogene, fusiforme Auftreibung des betroffenen Nervenabschnittes charakterisiert mit hypertrophhypoechogenen Faszikeln oder kompletter Maskierung der normalen Echotextur [127]. Sie erinnern damit an ein Kontinuitätsneurom (s.o.), was sich aber durch die Anamnese sicher abgrenzen lässt. Ähnliche sonografische Veränderungen beobachtet man bei fokalen Manifestationen von erworbenen demyeliniserenden Immunneuropathien (MMN, MADSAM, - Abb. 6). Differenzialdiagnostische Schwierigkeiten lassen sich nach der Erfahrung des Autors durch die Einbeziehung der Elektrodiagnostik (Leitungsblock, abnorme temporale Dispersion über dem betroffenen Nervenabschnitt), durch den sonografischen Nachweis eines multifokalen Vorkommens und schließlich durch die fehlende oder kaum vorhandene KM-Aufnahme in der 3T-MR-Neurografie (fettgesättigten T1-gewichteten Sequenzen nach sonografischer Markeriung) vermeiden [127-129]. Darüber hinaus findet man in der einschlägigen Fachliteratur der letzten Jahre durch die verbesserte bildgebende Diagnostik immer mehr kasuistische Beiträge oder kleine Studien über Neoplasien nichtneuralen Ursprungs, die sonografisch plexiformen Tumoren ähneln und sich in der MR-Neurografie zum Teil auch durch eine Kontrastmittelaufnahme auszeichnen. Die definitive Diagnose gelingt hier meist nur durch die Biopsie eines (funktionslosen) Faszikels [128,129]. Beispiele sind primär intraneurale Lymphome sowie auch sekundäre extramedulläre Manifestationen eines Plasmozytoms [130-134]. Wir selbst und andere haben Fälle eines intraneuralen Sarkoidosis-Lymphoma Syndroms, ein Amyloidom (nicht zu verwechseln mit der systemischen Amyloidose) und eine intraneurale Metastase eines Mamma-Karzinoms beobachtet [135-139]. Abschließend soll noch das intraneurale Nervenscheidenganglion genannt werden, welches häufig die benachbarten Nervenstämme des Kniegelenkes befällt (N. ischiadicus, N. peroneus, N. tibialis), prinzipiell aber überall auftreten kann [140]. In 16\% der Fälle soll es Ursache einer nichttraumatischen Fibularisparese sein [92]. Ein solches Ganglion entwickelt sich durch retrograden Transport von Synovia durch den sensiblen Nervenast, welcher das benachbarte Gelenk versorgt, in den proximalen Nervenstamm. Ursächlich sind degenerative Veränderungen. Das erklärt auch die fluktuierende Klinik, welche von den Druckverhältnissen im Gelenk bestimmt wird. Durch steigenden Druck gelangt immer mehr Synovia unter das Epineurium des proximalen Nervenstammes und erweitert diesen perlschnurartig. Die Faszikel mit Perinerium sind von der hypoechogenen Synovialflüssigkeit umgeben und werden durch diese komprimiert. Die chirurgische Therapie muss vor allem daraufhin abzielen, die Quelle des pathologischen synovialen Transports (sensibler Gelenkast) auszuschalten und darf nicht allein in einer Dekompression des betroffenen Nervs bestehen, da sonst Rezidive vorprogrammiert sind [141]. 


\section{Anwendungsmöglichkeiten bei Polyneuropathien} $\nabla$

In den letzten 4 Jahren ist die Zahl der Studien, welche sich mit der sonografischen Diagnostik von Polyneuropathien beschäftigen, deutlich gestiegen. Außerdem wurden Derivate der Nervenquerschnittsfläche eingeführt, um regionale Verteilungsmuster besser herausarbeiten zu können. Diese haben bisher allerdings noch nicht allgemeinen Eingang in die klinische Routine gefunden $[46,47,142]$. Bisher gibt es keine evidenzbasierten Empfehlungen zu Untersuchungsprotokollen. Das Screening der Nervenwurzeln des Plexus brachialis sowie der Nerven der oberen Extremität (Oberarm) ist besonders ergiebig bei demyelinisierenden Polyneuropathien. Diese weisen, ob hereditär oder erworben, die deutlichsten sonografischen Veränderungen auf $[142,143]$. Sie sind bei der CMT-1 besonders ausgeprägt, wobei sich diffuse Vergrößerungen der Nervenquerschnittfläche und Faszikel auch außerhalb der anatomischen Engpässe nachweisen lassen. Die Vergrößerung der Nervenquerschnittfläche übersteigt hier meist das Doppelte des jeweiligen Normwertes [142,143]. Bei der HNPP hingegen finden sich diese Hypertrophien multipel und fokal betont an (auch asymtomatischen) anatomischen Engpässen [144]. Erworbene demyeliniserende Neuropathien (AIDP, CIDP, MMN) sind durch keine, wenige oder fokal betonte Nerven- und Faszikelhypertrophien charakterisiert, wobei die Vergrößerung der Nervenquerschnittsfläche im Vergleich zu den herditären Formen deutlich geringer ausfällt $[142,143]$. Mehrfach wurde über die Korrelation der fokalen sonografischen Veränderungen zur Lokalisation von elektrophysiolgisch nachweisbaren Leitungsblöcken bei CIDP berichtet [145-156]. Verwendet man 4 Screening-Messstellen an der oberen Extremität, kann aufgrund der o.g. Merkmale eine Differenzierung zwischen CMT-1 und CIDP mit einer Sensitivität von $90 \%$ und einer Spezifität von 94\% erfolgen [142]. Eine ähnliche Methode verwendeten KERASNOUDIS et al. zur Differenzierung zwischen AIDP und CIDP (Bochum ultrasound score - BUS, Sensitivität/Spezifität: 80/100\%) [147]. Auch bei der MADSAM sind fokale Nervenhypertrophien an der Stelle von Leitungsblöcken und nicht mehr bestehenden Leitungsblöcken nachgewiesen worden [148]. Ähnlich wie bei den publizierten MADSAM-Fällen fehlte in mehreren neuen Studien über demyeliniserende Immunneuropathien der Zusammenhang zwischen dem Grad der sonografischen Veränderungen und der Klinik und die Korrelation zu elektrophysiologischen Auffälligkeiten war nur gering [73-75]. In einer kürzlich publizierten retrospektiven Arbeit nahmen die sonografischen Auffälligkeiten bei 23 Patienten mit CIDP unter immunsuppressiver Therapie mit klinischer Remission ab oder normalisierten sich vollständig [149]. Deutlich weniger Arbeiten wurden bisher über die zahlenmäßig viel häufigere diabetische Neuropathie publiziert. Einige Autoren fanden eine oberhalb vom Malleolus medialis signifikant vergrößerte Nervenquerschnittsfläche des N. tibialis (Cut-Off: $19 \mathrm{~mm}^{2}$, Sensitivität/Spezifität: 69/77\%), während andere an rein sensiblen Nerven (N. suralis, N. peroneus superficialis Höhe Sprunggelenk) keine signifikanten Unterschiede zu gesunden Kontrollen feststellen konnten [150]. Allerdings zeigten sich unter Verwendung einer $22 \mathrm{MHz}$-Sonde in einer Vorläuferstudie verdickte Faszikel des N. suralis bei Typ II Diabetikern mit Neuropathie [151]. Auch an der oberen Extremität wurde vereinzelt über signifikant größere Nervenquerschnittsflächen bei Patienten mit diabetischer Polyneuropathie berichtet [73]. Darüber hinaus existieren Berichte über akute und multifokale Nervenhypertrophien am N. medianus, ulnaris und tibialis bei systemischer Vaskulitis, die sich zum Teil nach Kortikosteroidthetrapie zurückbildeten [152-154]. Bezüglich der Polyneuropathien anderer Genese ist die Datenlage schwach. Zusammenfassend eignet sich die Sonografie als diagnostisches Zusatzkriterium besonders zum Nachweis von hereditären oder erworbenen demyelinisierenden Polyneuropathien bzw. zur Abgrenzung gegenüber Mononeuropathien durch den Nachweis von fokalen, multifokalen oder generalisierten Nervenhypertrophien (auch) außerhalb der typischen anatomischen Engpässe. Ein Beispiel wird in 0 Abb. 6 gezeigt.

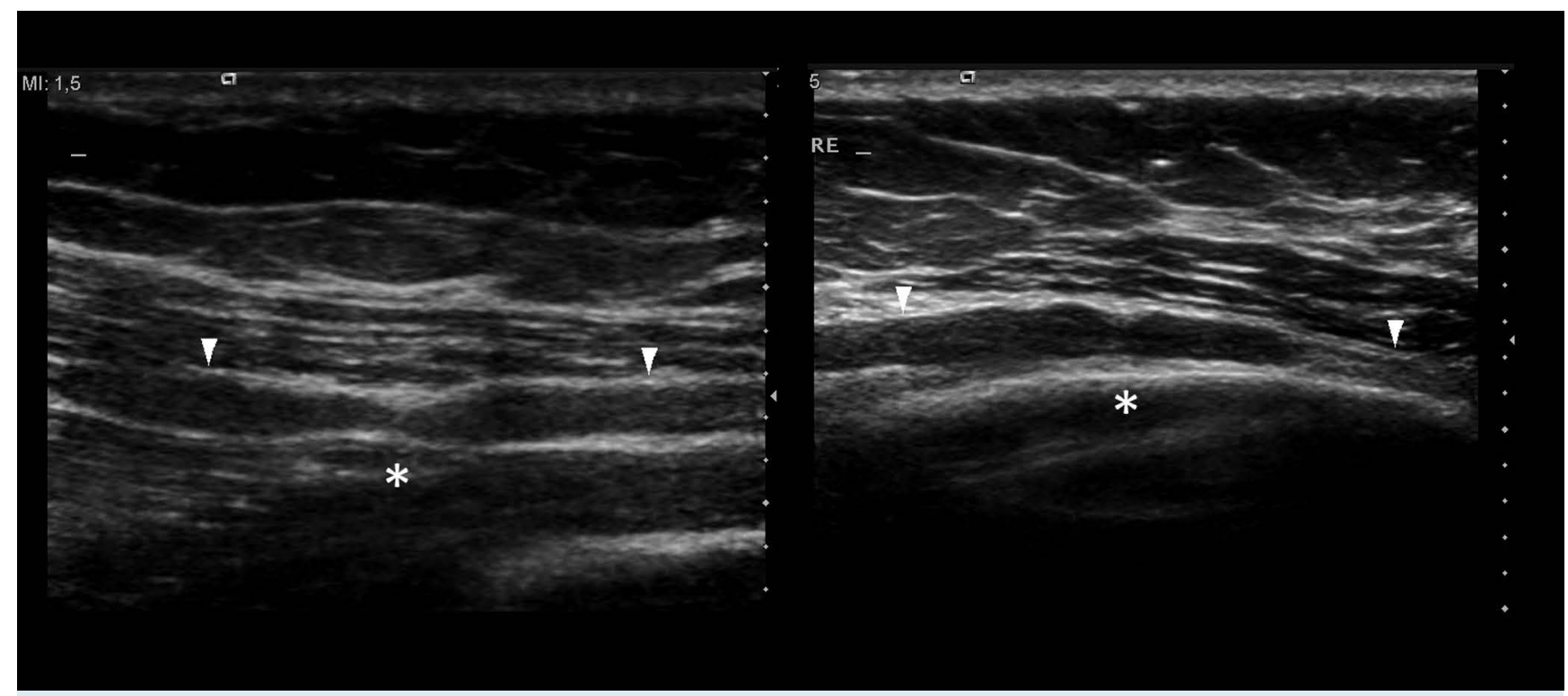

Abb. 7 Linker Teil: Nerventorsion des N. radialis communis am Oberarm. Beachte das sanduhrförmige Aussehen mit hyperechogener Striktur (Stern). 43-jähriger Patient mit „unklarer“ Radialisparese mehrere Jahre nach 2 identischen Attacken wie bei Parsonage-Tuner-Syndrom. Rechter Teil: 31-jährige Patienten mit 5 identischen, rezidivierenden Attacken eines Parsonage-Tuner-Syndroms am rechten Oberarm sowie progredienter Parese des N. radialis communis (Pfeilköpfe). Klinisch V.a. hereditäre Form (SEPT-9 Gendefekt). Beachte die Einziehung im Nerven (Stern). Mögliche Vorstufe einer Torsion? 


\section{Verschiedenes}

ALS: Patienten mit Amyotropher Lateralsklerose zeigten mehr in den cervicalen Nervenwurzeln als in den peripheren Nerven eine signifikante Verminderung der Nervenquerschnittsfläche im Vergleich zu gesunden Kontrollen, worin sich wahrscheinlich die axonale Ausdünnung widerspiegelt [155]. Darüber hinaus konnte durch Untersuchung der Muskulatur eine Zunahme der Echogenität durch Denervierung und bindegewebigen Umbau nachgewiesen werden. Außerdem lassen sich Faszikulationen auch in tief gelegenen Muskeln mit hoher Sensitivität detektieren [156].

Spontane Nerventorsionen: Vor mehr als 20 Jahren erschienen erste Berichte über spontane (nicht traumatisch bedingte) Nerventorsionen [157]. Die genaue Ätiologie ist bis heute nicht geklärt. Allerdings mehren sich die Hinweise auf eine entzündliche Genese [158]. Zum einen ist die Anamnese völlig identisch mit der eines Parsonage-Turner-Syndroms (Armplexusneuritis). Die Patienten klagen initial über reißenden Schmerzen in Projektion auf das betroffene Nervensegment. Darauf folgen graduell unterschiedlich ausgeprägte Paresen [159]. Zweites fanden man in Nervenbiopsien von Patienten, die sich einer Operation unterzogen hatten (Resektion mit Graft) CD8-positive T-Lymphozyten-Infiltrationen [158]. Einige Autoren differenzieren die graduell unterschiedlich ausgeprägte Konstriktion von der völligen Torsion des gesamten Nerven oder einzelner Faszikel [160]. Sie können einzeln aber auch in Serie vorkommen [161]. Am häufigsten wurden Nerventorsionen am N. radialis communis, $\mathrm{N}$. interosseous anterior und posterior sowie seltener am N. axillaris und N. suprascapularis beobachtet [159]. Die meisten dieser Nerven befinden sich in der Nähe des Ellenbogengelenkes. Es wird darüber spekuliert, dass nach einer lokalen Entzündung die Steifigkeit der Faszikel im Sinne eines Mikro-Kompartment-Syndroms zunimmt, sodass transversal verlaufende Strukturen wie Gefäße oder Faszien fixierend oder zusammenziehen wirken können. Wiederholte Rotationsbewegungen im Ellenbogengelenk unterstützen möglicherweise diesen Prozess [159]. Sonografisch ist eine Torsion durch ein urglasähnliches Aussehen des betroffenen Nervensegmentes charakterisiert mit meist hypoechogener proximaler mehr als distaler Kongestion ohne Hypervaskularisation. $\triangle$ Abb. 7 zeigt ein Beispiel. In einer größeren Studie konnten durch Ultraschall alle Torsionen/Konstriktionen detektiert werden $[161,162]$. Spezielle diffusionsgewichtete 3T-MRN Sequenzen sind ebenfalls geeignet [161]. Die Erkennung dieser Entität als mögliche Komplikation eines PARSONAGE Turner Syndroms ist wichtig, da in vielen Fällen eine chirurgische Therapie $\mathrm{zu}$ einer teilweisen oder vollständigen Wiederherstellung der Nervenfunktion führen kann. In aktuellen Studien orientierte sich diese an dem Schweregrad der Veränderungen beginnend mit einer Derotation sowie interfaszikulären Neurolyse in leichten Stadien bis hin zur Neurorraphie oder Segmentresektion mit Graft $[158,163,164]$.

Interessenkonflikt: Der Autor erhielt Vortragshonorar von Toshiba Medical Systems.

\section{Literatur \\ $\nabla$}

Die zitierte Literatur finden Sie im Internet unter http://10.1055/ s-0035-1549945 